\title{
Influência do estresse nos hábitos alimentares de estudantes do primeiro ano do curso de Medicina de uma universidade pública
}

\author{
Stress influence on eating habits of first year Medicine students at a public university \\ Influencia del estrés en los hábitos alimentarios de los estudiantes de primer año de Medicina de \\ una universidad pública
}

\author{
Ariane Araújo de Souza \\ ORCID: https://orcid.org/0000-0001-9592-2881 \\ Universidade Estadual do Ceará, Brasil \\ E-mail:ariane.araujo@aluno.uece.br \\ Maria das Graças Barbosa Peixoto \\ ORCID: https://orcid.org/0000-0002-9800-1778 \\ Universidade Estadual do Ceará, Brasil \\ E-mail: maria.gracas@uece.br \\ Francisco Régis da Silva \\ ORCID: https://orcid.org/0000-0001-5470-2874 \\ Universidade Estadual do Ceará, Brasil \\ E-mail: regisfrs.silva@uece.br \\ Claúdia Machado Coelho Souza de Vasconcelos \\ ORCID: https://orcid.org/0000-0002-3395-6143 \\ Universidade Estadual do Ceará, Brasil \\ E-mail: claudia.vasconcelos@uece.br \\ Francisco José Maia Pinto \\ ORCID: https://orcid.org/0000-0003-2976-7857 \\ Universidade Estadual do Ceará, Brasi \\ E-mail: maia.pinto@yahoo.com.br
}

\begin{abstract}
Resumo
Objetivo: Analisar a influência do estresse nos hábitos alimentares dos estudantes universitários. Métodos: Trata-se de estudo transversal realizado em uma universidade pública, envolvendo acadêmicos do primeiro ano do curso de Medicina, no período de novembro de 2020 a julho de 2021, dos quais 62 participaram, constituindo, assim, a amostra. Foi realizada coleta de dados através de um questionário on-line contendo 33 questões, produzido na ferramenta Google Forms. As variáveis investigadas foram: variáveis associativas (avaliação sociodemográfica e consumo alimentar) e desfecho (estresse). Foram realizadas análises descritiva e inferencial dos dados obtidos, utilizando-se testes estatísticos para estabelecer relações entre as variáveis. Resultados: Verificou-se que a maioria possuía idade abaixo dos 24 anos, vivia sem companheiro(a), tinha renda familiar maior que três salários-mínimos e apresentava IMC < 25. Além disso, a maioria consumia frutas ou copos de suco de frutas, verduras, legumes, leguminosas, proteína animal, frituras, embutidos, guloseimas, carboidratos em quantidade excessiva ou insuficiente, assim como consumia carne de gado ou frango com gordura ou não consumia carne, utilizava óleo vegetal, não acrescentava sal a mais em suas refeições e não costumava trocar almoço ou jantar por lanches. Observou-se que 32 participantes consideraram-se estressados. Na análise inferencial, pelo teste de Qui-quadrado, houve significância estatística apenas para a variável estresse e o consumo de frituras, embutidos e guloseimas. Conclusões: O estresse possui influência significativa nos hábitos alimentares dos estudantes universitários, que passam a consumir alimentos ricos em carboidratos e sódio, sendo os principais tipos de alimentos consumidos: frituras, embutidos e guloseimas.
\end{abstract}

Palavras-chave: Estresse; Hábitos alimentares; Estudantes universitários.

\begin{abstract}
Objective: Analyzing the stress influence on eating habits of university students. Methods: This work purposes a transversal study performed in a public university, involving first-year medicine academics, in the period from November 2020 to July 2021, defining a sample of 62 participants. Data collection was performed through an online questionnaire, produced in the Google Forms tool, containing 33 questions. The investigated variables were: associative variables (sociodemographic evaluation and food consumption) and outcome (stress). Descriptive and inferential analyses were executed from data obtained using statistical tests to establish relations between the variables. Results: The analyzes showed the majority were under 24 years old, resided without a partner, had a family income greater than three basic salaries, and presented BMI $<25$. Moreover, the majority consumed fruits or fruit
\end{abstract}


juices glasses, greenery, vegetables, leguminous, animal protein, fried and embed foods, goodies, excessive or insufficient amounts of carbohydrates, as well as consumed beef or chicken meat with fat or did not consume meat, used vegetable oil, did not add more salt in meal and was not used to exchange the lunch or dinner for snacks. It was observed that 32 participants considered themselves stressed. In inferential analysis, from the chi-squared test, the statistical significance occurred only for the stress variable and the fried, embed and goodies consumption. Conclusions: Stress has a significant influence on university students eating habits, which start to consume foods rich in carbohydrates and sodium, as fried, embed and goodies.

Keywords: Stress; Eating habits; University students.

\section{Resumen}

Objetivo: Analizar la influencia del estrés en los hábitos alimentarios de los estudiantes universitarios. Métodos: Estudio transversal realizado en una universidad pública, en que participaron estudiantes de primer año de medicina desde noviembre de 2020 hasta julio de 2021, de los cuales 62 participaron, constituyendo la muestra. La recogida de datos se llevó a cabo mediante cuestionario on-line que contenía 33 preguntas, elaborado en herramienta Google Forms. Las variables investigadas fueron: variables asociativas (evaluación sociodemográfica y consumo de alimentos) y de resultado (estrés). Se realizaron análisis descriptivos y inferenciales de datos obtenidos, utilizando pruebas estadísticas para establecer relaciones entre las variables. Resultados: Se verificó que la mayoría tenía menos de 24 años, vivía sin pareja, tenía ingresos familiares superiores a tres salarios-mínimos y presentaba un IMC < 25. Además, la mayoría consumía fruta o vasos de zumo, verduras, legumbres, proteínas animales, fritos, embutidos, dulces, carbohidratos en cantidad excesiva o insuficiente, así como carne de vacuno o pollo con grasa o no consumía carne, utilizaba aceite vegetal, no añadía sal extra en sus comidas y no solía cambiar la comida por bocadillos. Se observó que 32 participantes se consideraban estresados. En análisis inferencial, mediante la prueba de Qui-quadrado, hubo significación estadística para la variable estrés y el consumo de fritos, embutidos y dulces. Conclusiones: El estrés influye notablemente en los hábitos alimentarios de los estudiantes universitarios, que empiezan a consumir alimentos ricos en carbohidratos y sodio, y los principales tipos de alimentos consumidos son: fritos, embutidos y dulces.

Palabras clave: Estrés; Hábitos alimentarios; Estudiantes universitarios.

\section{Introdução}

A obesidade tem atingido, nas últimas décadas, grandes proporções, sendo um grave problema de saúde pública mundial. Além de estar presente em diversas faixas etárias, possui causas multifatoriais, dentre elas, fatores genéticos, culturais e do desenvolvimento psicológico e familiar (Lüdtke, Silva, Peuker et al, 2018). No entanto, não se constitui um transtorno psiquiátrico em si, como antes era compreendida e, portanto, associada à manifestação somática de um distúrbio psicológico subjacente. Existe, porém, o hábito de se alimentar, quando problemas emocionais estão presentes, o que sugere a relação entre estes sintomas e a compulsão por alimentos calóricos e aumento de peso (Abeso, 2016).

A literatura indica que situações estressoras direcionam o desejo por alimentos com maior palatabilidade e essa mudança no comportamento alimentar é mais perceptível em estudantes universitários, especialmente no início do curso, devido à mudança no estilo de vida. Tal mudança predispõe a desequilíbrios emocionais e comportamentais, e estes, nas escolhas alimentares (Penaforte, Matta \& Japur, 2016).

De acordo com a Organização Mundial da Saúde, o estresse atinge cerca de $90 \%$ da população mundial, sendo em torno de $70 \%$ referente aos brasileiros (WHO, 2000). Neste contexto, a exposição aguda ou crônica à tensão induz à ativação do eixo hipotálamo-hipófise-adrenal, aumentando a síntese de glicocorticoides, que podem aumentar o apetite, a ingestão de alimentos e o aumento de peso corporal (Ulrich-Lai, Fulton, Wilson et al, 2015).

Dentre os estudantes de ensino superior, o estresse entre acadêmicos do Curso de Medicina se torna preocupante, pois, além de afetar várias funções fisiológicas, psicológicas e cognitivas, prejudica ainda, a qualidade de vida e o cuidado dos mesmos com os pacientes e usuários dos serviços de saúde (Lima, Soares, Prado et al, 2016). Diante do exposto, a compreensão dos fatores que geram situações de tensão e estresse na rotina acadêmica e sua associação com a mudança de padrões alimentares torna-se fundamental, a fim de melhorar a qualidade de vida dos alunos.

O ingresso no ensino superior é, para muitos, um período de transição entre a vida escolar e a vida laboral, o que 
necessita de atenção, visto que os estudantes se deparam com dificuldades de adaptação, novos relacionamentos, exigências acadêmicas, perspectivas sobre o futuro da carreira e outras fontes de estresse, afetando a saúde mental caso não haja intervenção (Santos, Sousa, Rodrigues et al, 2019). O interesse por esta pesquisa surgiu pelo fato de a pesquisadora ser acadêmica do Curso de Medicina e percebera dificuldade encontrada por alunos em se adaptar ao ritmo acelerado de tarefas de ensino cobradas no curso e no controle do estresse, em sua vida diária.

O presente estudo teve como objetivo analisar a influência do estresse nos hábitos alimentares dos estudantes universitários.

\section{Metodologia}

Trata-se de um estudo do tipo transversal, descritivo e analítico, sendo útil para avaliar as necessidades de saúde de um grupo específico (Bonita, Beaglehole \& Kjellström, 2006), realizado em uma universidade pública de Fortaleza-CE, no período de novembro de 2020 a julho de 2021.

Fizeram parte da amostra deste estudo 62 acadêmicos devidamente matriculados e frequentando o primeiro ano do Curso de Medicina da Universidade Estadual do Ceará (UECE).

A coleta de dados foi realizada por meio de um questionário on-line produzido na ferramenta Google Forms e enviado via e-mail pessoal dos acadêmicos, a partir de lista disponibilizada pela coordenação do Curso de Medicina. Essa ferramenta permite a coleta de informações de forma simples e rápida, além de otimizar a análise dos dados da pesquisa. Os estudantes do primeiro ano (primeiro e segundo semestres) foram escolhidos devido ao fato de eles estarem sujeitos, de forma mais perceptível, às mudanças do estilo de vida ao adentrarem no ensino superior e, assim, predispostos às mudanças em seus hábitos alimentares.

As variáveis do estudo referiram-se ao desfecho, representado pela influência do estresse nos hábitos alimentares, dicotomizado em maior estresse e menor estresse. Em relação às variáveis associativas, as respostas foram dicotomizadas $(0$ : exposto; 1: não exposto).

A avaliação do estresse foi realizada por meio da Escala de Estresse Percebido (Perceived Stress Scale - PSS-14) para estimar o grau no qual os indivíduos percebem as situações como estressantes, através da escala validada para a língua portuguesa por Luft (2007) composta por 14 itens. As possíveis variáveis associativas referiram-se à avaliação sociodemográfica e ao consumo alimentar, totalizando 33 questões. O questionário semiestruturado sociodemográfico, elaborado pelos próprios pesquisadores, foi formado por nove questões, que contemplaram, além do semestre, as seguintes variáveis: sexo, idade, peso e altura (utilizados para o cálculo do Índice de Massa Corporal - IMC), estado civil, procedência, renda familiar e graduação prévia. A categoria relacionada ao consumo alimentar foi realizada por meio da adaptação do questionário estruturado, proposto pelo Ministério da Saúde (Brasil, 2008; Pomini, Sant'ana \& Souza, 2009), composto por 10 perguntas referentes ao consumo de alimentos. Para interpretação, utilizou-se a quantidade de porções determinadas pelo Departamento da Agricultura dos Estados Unidos da América, ao avaliar o consumo e a frequência da ingesta de alimentos contendo carboidratos, proteínas e gorduras.

Os dados obtidos por meio do questionário foram atrelados à planilha do Google e armazenados no programa Microsoft Office Excel, versão 13.0. Para análise e tratamento dos dados, utilizou-se o programa estatístico Statistical Package for the Social Sciences (SPSS), versão 23.0, a fim de verificar a existência de associação entre o desfecho e as variáveis associativas. Na análise descritiva, os dados foram interpretados por meio das frequências (absoluta e percentual), e a análise inferencial envolveu a associação entre as variáveis desfecho (estresse) e exploratórias (fatores sociodemográficos e nutricionais). $\mathrm{Na}$ análise inferencial, utilizou-se o teste Qui-quadrado de Pearson ao nível de significância de 5\%, com 
intervalo de confiança de $95 \%$.

A pesquisa foi aprovada pelo Comitê de Ética em Pesquisa da Universidade Estadual do Ceará, sob o Parecer Consubstanciado ${ }^{\circ} 4.488 .694$.

\section{Resultados}

As características gerais dos participantes por fatores sociodemográficos encontram-se na Tabela 1 e os fatores nutricionais são apresentados na Tabela 2.

Dos 62 estudantes que participaram da pesquisa, a idade média foi de 21,3 anos e a média de IMC, de $23,2 \mathrm{~kg} / \mathrm{m}^{2}$.

Em relação às características sociodemográficas, verificou-se que: a maioria estava na faixa etária abaixo dos 24 anos (52; 83,9\%), vivia sem companheiro(a) $(60 ; 96,8 \%)$, tinha renda familiar maior que três salários-mínimos $(36 ; 58,1 \%)$ e apresentava IMC $<25(43 ; 69,4 \%)$ (Tabela 1$)$.

Tabela 1: Características gerais dos participantes por fatores sociodemográficos, Fortaleza - CE, 2020.

\begin{tabular}{lll}
\hline Variáveis & N & \% \\
Sexo & 26 & 41,9 \\
Feminino & 36 & 58,1 \\
Masculino & & \\
Idade & 10 & 16,1 \\
$\geq 24$ anos & 52 & 83,9 \\
<24 anos & & \\
Estado civil & 2 & 3,2 \\
Com companheiro(a) ou divorciado(a) & 60 & 96,8 \\
Sem companheiro(a) & & \\
Renda familiar (s.m.) & 26 & 41,9 \\
Até 3 & 36 & 58,1 \\
$>3$ & & 30,6 \\
IMC & 19 & 69,4 \\
$\geq 25$ & 43 & \\
\hline 25 & & \\
\hline
\end{tabular}

s.m.: salário-mínimo.

Fonte: Autores (2021).

Em relação ao consumo alimentar dos participantes, observou-se que mais da metade dos estudantes consumia frutas ou copo de suco de frutas por dia $(56 ; 90,3 \%)$, verduras e legumes por dia $(52 ; 83,9 \%)$, leguminosas por dia $(60 ; 96,8 \%)$, não consumia carboidratos ou os consumia a partir de 6 colheres por dia $(34 ; 54,8 \%)$, consumia proteína animal por dia, de pelo menos dois pedaços/ovos $(51 ; 82,3 \%)$, consumia carne de gado ou frango com gordura ou não consumia carne $(41 ; 69,4)$, consumia frituras, embutidos e guloseimas pelo menos duas vezes por semana $(39 ; 62,9 \%)$, utilizava óleo vegetal $(42 ; 67,7 \%)$, não acrescentava sal a mais em suas refeições (57; 91,9\%) e não costumava trocar o almoço ou o jantar por lanches (38; $61,3 \%)$. Em relação ao estresse, verificou-se que a maioria considerou-se estressada (32; 51,6\%) (Tabela 2). 
Tabela 2: Consumo alimentar e estresse dos participantes, Fortaleza - CE, 2020.

\begin{tabular}{|c|c|c|}
\hline Variáveis & $\mathbf{N}$ & $\%$ \\
\hline \multicolumn{3}{|l|}{ Frutas ou copos de suco por dia } \\
\hline Não consome & 6 & 9,7 \\
\hline$\geq 1$ & 56 & 90,3 \\
\hline \multicolumn{3}{|l|}{ Verduras e legumes por dia } \\
\hline Não consome & 10 & 16,1 \\
\hline$\geq 1$ & 52 & 83,9 \\
\hline \multicolumn{3}{|l|}{ Leguminosas por dia } \\
\hline Não consome & 2 & 3,2 \\
\hline$\geq 1$ & 60 & 96,8 \\
\hline \multicolumn{3}{|l|}{ Carboidratos por dia } \\
\hline Não consome / consome a partir de 6 colheres & 34 & 54,8 \\
\hline De 1 a 5 colheres & 28 & 45,2 \\
\hline \multicolumn{3}{|l|}{ Proteína animal por dia } \\
\hline$<2$ pedaços/ovos & 11 & 17,7 \\
\hline$\geq 2$ pedaços/ovos & 51 & 82,3 \\
\hline \multicolumn{3}{|l|}{ Carne de gado ou frango com gordura } \\
\hline Sim / não consome carne & 41 & 69,4 \\
\hline Consome sem gordura & 19 & 30,6 \\
\hline \multicolumn{3}{|l|}{ FEG por semana } \\
\hline$\geq 2$ vezes & 39 & 62,9 \\
\hline$<2$ vezes & 23 & 37,1 \\
\hline \multicolumn{3}{|l|}{ Gordura utilizada } \\
\hline Margarina / gordura animal / vegetal & 20 & 32,3 \\
\hline Óleo vegetal & 42 & 67,7 \\
\hline \multicolumn{3}{|l|}{ Uso de sal a mais nas refeições } \\
\hline Sim & 5 & 8,1 \\
\hline Não & 57 & 91,9 \\
\hline \multicolumn{3}{|c|}{ Costume de trocar o almoço ou o jantar por lanches } \\
\hline Sim / às vezes & 24 & 38,7 \\
\hline Não & 38 & 61,3 \\
\hline \multicolumn{3}{|l|}{ Estresse dos participantes } \\
\hline Sim & 32 & 51,6 \\
\hline Não & 30 & 48,4 \\
\hline
\end{tabular}

FEG: frituras, embutidos e guloseimas.

Fonte: Autores (2021).

$\mathrm{Na}$ análise inferencial, verificou-se, pelo teste Qui-quadrado de Pearson, que houve significância estatística apenas para a variável estresse e o consumo de frituras, embutidos e guloseimas ( $\mathrm{p}=0,010$ ) (Tabela 3). No entanto, verificou-se não existência de associação entre estresse com estado civil $(\mathrm{p}=0,963)$ e estresse com consumo de leguminosas $(\mathrm{p}=0,963)$. 
Tabela 3: Associação entre as variáveis estresse e consumo de frituras, embutidos e guloseimas, Fortaleza - CE, 2020.

\begin{tabular}{ll}
\hline Consumo de FEG e estresse & p-valor $^{1}$ \\
$\geq 2$ vezes & 0,010 \\
$<2$ vezes & \\
\hline
\end{tabular}

FEG: frituras, embutidos e guloseimas.

1: Qui-quadrado de Pearson.

Fonte: Autores (2021).

\section{Discussão}

De acordo com os resultados obtidos com a análise dos dados, percebeu-se que a frequência de estresse na população estudada foi indicada pela maioria dos participantes, porém não esteve associada à maioria dos hábitos alimentares avaliados. No entanto, estes estudantes estressados consumiam frituras, embutidos e guloseimas no mínimo duas vezes por semana, o que indica que o estresse influência de forma significativa o consumo desse tipo de alimento (Ulrich-Lai, Fulton, Wilson et al, 2015). Isso pode ser explicado pelo fato de tais alimentos possuírem maior palatabilidade, trazendo satisfação ao consumo em situações de estresse.

Nos países industrializados, pesquisas mostram predisposição do sexo feminino ao estresse no ambiente de trabalho (Ludermir, 2000). Fatores psicossociais maiores em mulheres podem indicar aumento dos sintomas físicos e psicológicos devido à instabilidade emocional, diminuição da resiliência e comportamento social inadequado (Areias \& Guimarães, 2004). Nesta pesquisa, não foi possível associar estresse ao sexo, o que pode ser explicado pelo número menor de participantes do sexo feminino.

Em estudo nacional realizado com dados coletados de 13 capitais brasileiras, no período compreendido entre 2010 e 2013, com o objetivo de traçar o perfil de saúde das gerações, mostrou que a população na faixa etária de 24 a 37 anos era a mais estressada e mais ansiosa, em comparação à com idade abaixo dos 24 anos. É possível relacionar tal achado ao aumento da pressão vivenciado pelos jovens com o início da vida profissional (Lopes \& Alves, 2015). Entretanto, de acordo com a análise bivariada, não foi possível associar a idade dos participantes ao estresse, visto que a faixa etária não mostrou associação estatística significativa com a presença de estresse. Isso pode ser explicado pelo fato de a maioria dos estudantes que inicia o Curso de Medicina ser composta por indivíduos com idade abaixo de 24 anos.

Em relação ao estado civil, a literatura aponta que o maior índice de estresse se encontra em pessoas que convivem ou já conviveram com algum parceiro(a) (Rodrigues \& Ferreira, 2011), postulando que a quantidade de papeis assumidos, associa-se aos maiores níveis de estresse (Sadir, Bignotto \& Lipp, 2010). No entanto, neste estudo não houve significância estatística entre tais variáveis, o que pode ser explicado pelo fato de $96,8 \%$ da população estudada ser composta por pessoas solteiras.

Com relação à renda familiar, pesquisas mostram que, quanto maior a faixa salarial, menor a suscetibilidade ao estresse e aos transtornos psicológicos, assim como, existe a melhor percepção do suporte familiar, indicando que a renda é fator de proteção àquele (Camargo, Calais \& Sartori, 2015). Neste estudo a porcentagem de estudantes estressados com renda de até três salários-mínimos foi menor que a dos participantes com renda superior a três salários, não sendo estabelecida associação significativa para corroborar os dados da literatura.

$\mathrm{O}$ aumento do consumo de alimentos palatáveis, associados à alimentação emocional e ao descontrole alimentar, pode estar relacionado à resposta fisiológica ao estresse e à consequente tentativa de melhora do humor (Penaforte, Matta \& Japur, 2016), fato que pode ser refletido no peso. Em um estudo de 2008 (Lessa \& Montenegro, 2008), realizado com acadêmicos do curso de Medicina de uma universidade alagoana, foi identificada prevalência de sobrepeso entre estes estudantes. No presente 
estudo, o excesso de peso foi visto em aproximadamente um terço dos participantes, mas a associação entre o estresse e o IMC não foi significativa para relacionar esse dado com a maior predisposição desse grupo àquele fator.

Uma pesquisa de 2010 realizada na cidade de Boston (Laugero, Falcon \& Tucker, 2011) avaliou possíveis associações entre o estresse e o padrão dietético em adultos. Verificou-se que aquele fator está relacionado ao elevado consumo de carboidratos, lipídios, refeições com alto teor de sódio e índice glicêmico aumentado, em detrimento do consumo de frutas, verduras, fibras e proteínas, como mostram outros estudos (Brunori, Lopes, Cavalcante et al, 2015; Ulrich-Lai, Fulton, Wilson et al, 2015). Entretanto, na avaliação alimentar dos participantes deste estudo, não foi possível associar o estresse ao maior consumo de frutas, verduras, legumes e leguminosas. Da mesma forma, o consumo de lipídios não teve uma associação significativa com o estresse. No entanto, a ingesta de frituras, embutidos e enlatados, alimentos compostos por altos índices de açúcares, sódio e lipídios, mostrou associação significativa com a presença de estresse, corroborando os achados da literatura.

Para Penaforte (2016), o padrão alimentar dos universitários é de baixa qualidade, tendo em vista a maior regularidade de consumo de alimentos ricos em açúcares em detrimento do consumo de frutas e vegetais. Estudantes universitários são, em sua maioria, os principais responsáveis por cuidar de sua alimentação. Somam-se a isto, a rotina atarefada e as exigências acadêmicas inerentes à vida universitária. Como consequência, as escolhas alimentares parecem se deslocar para alimentos de fácil acesso, baixo custo e preparo rápido.

Outros estudos apontam a existência entre a influência de transtornos afetivos, como depressão e ansiedade, nos hábitos alimentares de estudantes universitários da área da saúde (Blandón, León, Durango et al, 2016), porém, sobre o estresse em si, os mesmos ainda são incipientes, sendo de fundamental importância pesquisas que busquem avaliar a associação entre a influência do estresse na alimentação desse grupo.

Este estudo apresenta algumas limitações, pois a amostra escolhida foi não probabilística, por conveniência, relativa ao peso e a altura dos estudantes, que foram autoreferidos, tendo o risco de subestimação dos dados. Além disso, pode-se inferir que os estudantes do primeiro ano do Curso de Medicina considerados estressados estão mais suscetíveis ao consumo de carboidratos, frituras, embutidos e enlatados, bem como de dietas com alto teor de sódio, em consonância com os resultados encontrados em outras pesquisas.

\section{Conclusões}

Os achados obtidos neste estudo mostram que o estresse possui influência significativa em alguns aspectos dos hábitos alimentares dos estudantes universitários, que passam a consumir alimentos ricos em carboidratos e sódio. Além disso, a quantidade significativa de estudantes com estresse e com excesso de peso configura-se em problema de saúde pública, devendo essa relação ser aprofundada em pesquisas futuras, pois há indícios de que esse agravante pode levar a piores hábitos alimentares.

É preciso destacar a necessidade de se estudar mais esse assunto de maneira longitudinal para que sejam estabelecidas outras possíveis interferências do estresse nas escolhas alimentares da população universitária, pois a existência dessa relação pode acometer a saúde do indivíduo como um todo e seu desempenho acadêmico. Da mesma forma, torna-se fundamental ampliar as abordagens metodológicas, para que haja uma investigação mais aprofundada acerca dos fatores que estão envolvidos na gênese desse problema, não somente com abordagens quantitativas, mas também qualitativas.

Portanto, os resultados apresentados no presente estudo apontam a urgência de intervenções em saúde nesse grupo. As instituições de ensino superior devem estar preparadas para direcionar os alunos ao acompanhamento nutricional, por meio de ações de educação alimentar e nutricional, bem como ressaltar a importância da saúde mental dos mesmos, buscando desenvolver estratégias que ofereçam suporte adequado aos estudantes. 
Devido às limitações desta pesquisa, sugerem-se alguns aspectos que podem ser trabalhados em estudos futuros como: ampliar a amostra, permitindo uma melhor representação da população estudada; dar preferência à aplicação do questionário de forma presencial, aferindo as medidas antropométricas dos participantes, para que sejam evitados dados subestimados; e fornecer detalhes adicionais às questões, proporcionando melhor entendimento do objetivo da pesquisa e possibilidade de mais associações entre as variáveis envolvidas.

\section{Referências}

Areias, M. E. Q., \& Guimarães, L. A. M. (2004). Gênero e estresse em trabalhadores de uma universidade pública do Estado de São Paulo. Psicologia em Estudo, Maringá. 9(2), 255-262. https://www.scielo.br/j/pe/a/jytVTYD8fMqrGj8cJrFcSzQ/?lang=pt

Associação Brasileira para o Estudo da Obesidade e da Síndrome Metabólica - ABESO (2016). Diretrizes brasileiras de obesidade 2016. São Paulo, (4ª.ed.) https://abeso.org.br/wp-content/uploads/2019/12/Diretrizes-Download-Diretrizes-Brasileiras-de-Obesidade-2016.pdf.

Blandón, D. A. S., León, T. C., Durango, M. P. P., Tejada-Tayabas, L. M., \& Lucio, A. G. P. (2016). Ansiedad, depresión y actividad física asociados a sobrepeso/obesidad en estudiantes de dos universidades mexicanas. Revista Hacia la Promoción de la Salud, 21(2). https://doi.org/10.17151/hpsal.2016.21.2.8

Bonita, R., Beaglehole, R., \& Kjellström, T. (2010). Epidemiologia Básica. (2 ${ }^{\mathrm{a}} \quad$ ed.): Grupo Editorial Nacional. https://edisciplinas.usp.br/pluginfile.php/964545/mod_resource/content/1/Bointa\%20-\%20epidemiologia\%20basica.pdf

Brasil (2008). Ministério da Saúde. Secretaria de Atenção à Saúde. Guia alimentar para a população brasileira: promovendo a alimentação saudável. Série A. Normas $e$ Manuais Técnicos. Departamento de Atenção Básica. Brasília: Ministério da Saúde. https://www.nescon.medicina.ufmg.br/biblioteca/imagem/0290.pdf

Brunori, E. H. F. R., Lopes, C. T., Cavalcante, A. M. R. Z., Silva, M. C. S. R., Lopes, J. L., \& Barros, A. L. B. L. (2015). Dietary intake and stress in patients with acute coronary syndrome. Rev. Bras. Enferm. 68(5), 528-34. http://dx.doi.org/10.1590/0034-7167.2015680507i

Camargo, V. C. V., Calais, S. L., \& Sartori, M. M. P. (2015). Estresse, depressão e percepção de suporte familiar em estudantes de educação profissionalizante. Estudos de Psicologia, Campinas. 32(4), 595-604. http://dx.doi.org/10.1590/0103-166x2015000400003

Laugero, K. D., Falcon, L. M., \& Tucker, K. L. (2011). Relationship between perceived stress and dietary and activity patterns in older adults participating in the Boston Puerto Rican Health Study. Appetite. 56(1), 194-204. Elsevier BV. http://dx.doi.org/10.1016/j.appet.2010.11.001

Lessa, S. S., \& Montenegro, A. C. (2008). Avaliação da prevalência de sobrepeso, do perfil nutricional e do nível de atividade física nos estudantes de medicina da Universidade de Ciências da Saúde de Alagoas - UNCISAL. Rev. Soc. Bra. Clin. Med. 6(3), 90-93. http://files.bvs.br/upload/S/16791010/2008/v6n3/a90-93.pdf

Lima, R. L., Soares, M. E. C., Prado, S. N., \& Albuquerque, G. S. C. (2016). Estresse do Estudante de Medicina e Rendimento Acadêmico. Revista Brasileira de Educação Médica, Rio de Janeiro. 40(4), 678-684. https://www.scielo.br/scielo.php?script=sci_arttext\&pid=S0100-55022016000400678\&lng= pt\&nrm=iso

Lopes, M., \& Alves, G. (ed.). (2015). V Estudo Saúde Ativa: Gerações. Sulamérica $\quad$ Saúde. https://portal.sulamericaseguros.com.br/lumis/portal/file/fileDownload.jsp?fileId=8A61648C54A27C270154BFC4EB070A57\&inline=1

Ludermir, A. B. (2000). Inserção produtiva, gênero e saúde mental. Cadernos de Saúde Pública. 16(3), 123-129. https://www.scielo.br/j/csp/a/xYjF5HZbZQnYcDN579zb9Nm/?lang=pt

Lüdtke, L., Silva, B. M. B., Peuker, A. C. W. B., \& Garcia, E. L. (2018). Obesidade, depressão e estresse: relato de uma intervenção multidisciplinar em grupo com adolescentes. Revista Interdisciplinar de Promoção da Saúde, Santa Cruz do Sul. 1(4), 256-266. https://online.unisc.br/seer/index.php/ripsunisc/article/view/13078

Luft, C. B. D., Sanches, S. O., Mazo, G. Z., \& Andrade A. (2007). Versão brasileira da Escala de Estresse Percebido: tradução e validação para idosos. Revista de Saúde Pública. 41(4), 606-615. https://www.scielo.br/j/rsp/a/bgpXDHZXQXNqVS8JLnLdLhr/?lang=pt

Penaforte, F. R. O., Matta, N. C., \& Japur, C. C. (2016). Associação entre estresse e comportamento alimentar em estudantes universitários. Demetra: Alimentação, Nutrição e Saúde, Rio de Janeiro. 11(1), 225-237. https://www.e-publicacoes.uerj.br/index.php/demetra/article/view/18592

Pomini, R. Z., Sant'ana, D. M. G., \& Souza, M. C. C. (2009). Avaliação dos hábitos alimentares dos adolescentes de uma escola da rede de ensino da cidade de Umuarama - PR. Arquivos do MUDI, 13(1/2/3). https://periodicos.uem.br/ojs/index.php/ArqMudi/article/view/20017/10860

Rodrigues, V. M. C. P., \& Ferreira, A. S. S. (2011). Fatores geradores de estresse em Enfermeiros de Unidades de Terapia Intensiva. Rev. Latino-Am. Enfermagem. 19(4). https://www.scielo.br/j/rlae/a/jcFdMY9jMTj7hj7Nqxqdk3r/?lang=pt\&format=pdf

Sadir, M. A., Bignotto, M. M., \& Lipp, M. E. N. (2010). Stress e qualidade de vida: influência de algumas variáveis pessoais. Paidéia, Ribeirão Preto. 20(45), 73-81. http://dx.doi.org/10.1590/s0103-863x2010000100010

Santos, R. J. L. L., Sousa, E. P., Rodrigues, G. M. M., \& Quaresma, P. C. (2019). Estresse em acadêmicos de enfermagem: importância de identificar o agente estressor. Brazilian Journal Of Health Review, Luziânia. 2(2), 1086-1094. https://www.brazilianjournals.com/index.php/BJHR/article/view/1304/1183 
Research, Society and Development, v. 10, n. 11, e223101119609, 2021

(CC BY 4.0) | ISSN 2525-3409 | DOI: http://dx.doi.org/10.33448/rsd-v10i11.19609

Ulrich-Lai, Y. M., Fulton, S., Wilson, M., Petrovich, G., \& Rinaman, L. (2015). Stress exposure, food intake and emotional state. Stress. 18(4), 381-399. $10.3109 / 10253890.2015 .1062981$

World Health Organization. (2000). Obesity: preventing and managing the global epidemic: report of a WHO consultation. World Health Organization. https://apps.who.int/iris/handle/10665/42330 